\title{
The role of side chains in the interaction of new antitumor pyrimidoacridinetriones with DNA: Molecular dynamics simula- tions $^{\text {t* }}$
}

\author{
Jan Mazerski ${ }^{1 \bowtie}$, Ippolito Antonini ${ }^{2}$ and Sante Martelli ${ }^{2}$ \\ ${ }^{1}$ Department of Pharmaceutical Technology and Biochemistry, Technical University of Gdańsk, \\ G. Narutowicza 11/12, 80-952 Gdańsk, Poland; \\ ${ }^{2}$ Department of Chemical Sciences, University of Camerino, 62-032 Camerino (MC), Italy
}

Received: 25 October, 1999

Key words: pyrimidoacridinetrione antitumor agents, structure of intercalation complex, role of side chain(s), molecular dynamics simulations

\begin{abstract}
Pyrimidoacridinetriones (PATs) are a new group of highly active antitumor compounds. It seems reasonable to assume that, like for some other acridine derivatives, intercalation into DNA is a necessary, however not a sufficient condition for antitumor activity of these compounds. Rational design of new compounds of this chemotype requires knowledge about the structure of the intercalation complex, as well as about interactions responsible for its stability. Computer simulation techniques such as molecular dynamics (MD) may provide valuable information about these problems.

The results of MD simulations performed for three rationally selected PATs are presented in this paper. The compounds differ in the number and position of side chains. Each of the compounds was simulated in two systems: i) in water, and ii) in the intercalation complex with the dodecamer duplex d(GCGCGCGCGCGC) 2 . The orientation of the side chain in relation to the ring system is determined by the position of its attachment. Orientation of the ring system inside the intercalation cavity depends on the number and position of side chain(s). The conformations of the side chain(s) of all PATs studied in the intercalation complex were found to be very similar to those observed in water.
\end{abstract}

\footnotetext{
$\bar{\star}$ Presented at the $7^{\text {th }}$ International Symposium on Molecular Aspects of Chemotherapy, September 8-11, 1999, Gdańsk, Poland.

This work was supported in part by: the State Committee for Scientific Research (KBN, Poland) grant No. 3 T09A 124 14, Italian CNR, and Faculty of Chemistry, Technical University of Gdańsk (Poland) ${ }^{\star}$ To whom correspondance should be addressed
}

Abbreviations: PAT, pyrimidoacridinetrione; MD, molecular dynamics; C, and G: deoxynucleotides of cytosine, and guanine, respectively. 
The notable achievements in the field of development of antitumor drugs consisting of fully synthetic DNA-interacting agents of anthracenedione and acridine groups, such as mitoxantrone [1-5] and amsacrine [6, 7], respectively, have raised a great interest in the synthesis and modifications of these two classes of compounds. Studies on anthrapyrazoles [8, 9], pyrazoloacridines [10-11], benzothiopyranoindazoles [12], acridine carboxamides [13, 14], imidazoacridinones [15, 16], and triazoloacridinones [17] are the most representative examples of the research in this area. As part of a research project devoted to the investigation of new chemotypes of antitumor intercalating agents, recently we have reported [18-20] the synthesis and the antitumor properties of a series of mono- and di-alkylaminoalkylamino derivatives of pyrimido[5,6,1-de]acridine-1,3,7-triones (Scheme 1).

The mechanism of action of this new class of antitumor agents is not yet known. However, in analogy to anthracenediones, it seems reasonable to assume that also for PATs the intercalation to DNA is a necessary, but not a sufficient, condition for antitumor activity [21, 22]. It has been demonstrated that interactions of these compounds with DNA are strongly affected by the number and structure of side chain(s) [23]. Further rational design of new compounds of this chemotype requires knowledge about interactions between side chain(s) and DNA. Traditional approach is time consuming and demands large series of derivatives for structure-activity relationship
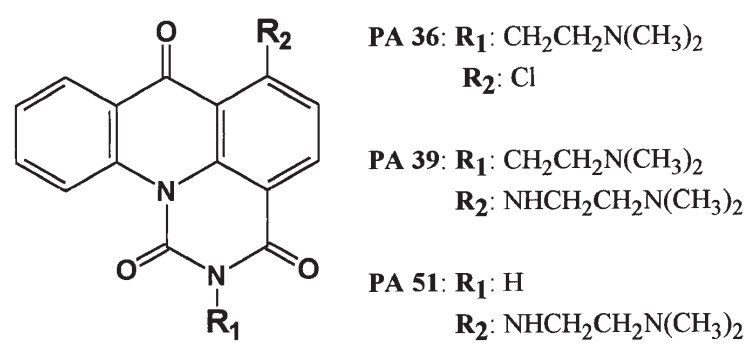

Scheme 1. Chemical structures of pyrimidoacridinetriones (PATs) studied. studies [2, 3] and/or extensive biochemical and biophysical work. Not disregarding this experimental approach, it seems reasonable to begin the analysis of this problem with modern computer simulation techniques such as molecular dynamics. Since the pioneering study of the dynamics of DNA in vacuum [24] molecular dynamics has now progressed to such an extent that specificity effects of drug-DNA interactions are solved [25]. Intercalation has been studied particularly extensively [26-30].

In this work we have analysed the structure and dynamics of a dodecamer duplex $\mathrm{d}(\mathrm{GCGCGC} \mid \mathrm{GCGCGC})_{2}$ with the use of three rationally selected PAT derivatives intercalated between central base-pairs 6C-19G/ 7G-18C. The selected compounds (Scheme 1) differ in the number and positions of side chain(s). In compound PA 36, a single dimethylaminoethyl chain is attached to nitrogen atom in position 2 in pyrimidoacridinetrione ring system while in PA 51 a dimethylaminoethylamino chain is attached to carbon atom in position 6 of the ring system. Compound PA 39 has chains in both positions and is characterised by a significantly higher ability of intercalation to DNA. We have focused particular attention on the orientation of intercalated molecules in the intercalation cavity, as well as on recognition of the nature of interactions responsible for stability of the complex. In our recent papers [30, 31] we have shown that molecular dynamics simulations could solve such problems.

\section{METHODOLOGY}

The dynamic simulations reported in this work were performed with GROMOS96, a set of molecular dynamics programs from the University of Groningen, Netherland [32], implemented on INDIGO 2 (SG) workstation.

The PATs were simulated as mono-, PA 36 and PA 51, or dication, PA 39, with protonated side chain amino group(s). The pe- 
riodic boundary condition was applied, all bond lengths were constrained to their equivalence values using SHAKE algorithm [33], and distances related to Watson-Crick hydrogen bonds were restrained to $0.21 \mathrm{~nm}$ during all simulations [34]. A dielectric constant of 1 and a cut-off of $0.8 \mathrm{~nm}$ were used for non-bonded interactions, whereas a cut-off of

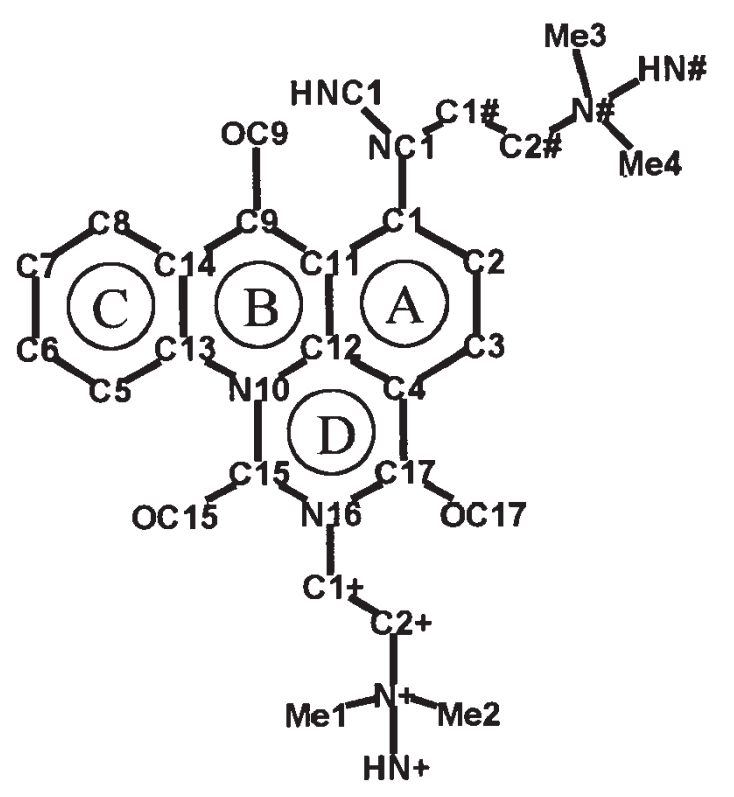

Scheme 2. Designations of PA 39 atoms used in the present work. For other PATs an analogous system was used.

$1.2 \mathrm{~nm}$ was used for long term electrostatic interactions. An appropriate number of counterions, 1 or $2 \mathrm{Cl}^{-}$for PATs and $22 \mathrm{Na}^{+}$ for the dodecamer of DNA, and of water molecules, about 1700 for PAT and about 1300 for the intercalation complexes, were added to the system. In order to save computation time the intercalation phenomena were analysed with the DNA fragment in which the intercalation cavity was pre-formed [30]. The initial co-ordinates of PATs were obtained from molecular modelling of the molecules by program INSIGHT II (MSI.). The dynamic trajectories were collected during 100 ps simulations. The detailed protocol of calculations is being published in the accompaying paper [31].

\section{RESULTS}

\section{PA 36}

A visual analysis of the dynamic trajectory of PA 36 in water (Fig. 1a), indicates that the ring system exhibits only small deviations from the mean plane. The $N, N$-dimethylaminoethyl side chain attached to the nitrogen atom N16 (Scheme 2) is of higher flexibility. However, the protonated, terminal nitrogen atom of this chain lies not very far from the mean plane of the ring system. It is interesting to note that this nitrogen atom does not participate in any intramolecular hydrogen bonds.

It is noteworthy that the shape of PA 36 molecule taking part in the intercalation complex, (Fig. 1b), is not significantly different from that in water environment.

The analysis of geometry of the intercalation complex revealed characteristic orientation of the PA 36 ring system in relation to the neighbor base-pairs (Fig. 2). The mean plane of the ring system is parallel to the mean planes of the neighbor base pairs. The long axis of the acridine ring system is nearly parallel to the direction of inter-base hydrogen bonds between $6 \mathrm{C}$ and $19 \mathrm{G}$. Location of rings $\mathrm{C}$ and $\mathrm{D}$ is the characteristic feature of this orientation. The terminal ring $\mathrm{C}$ is located in the major groove, whereas the additional 6-membered ring $\mathrm{D}$ is placed in the minor groove. The side chain lies in the mean plane of the ring system near one of the sides of the minor groove. A detailed analysis of interactions between the side chain and the dodecamer duplex (Fig. 3) has shown that the protonated, terminal amino group of the chain participates in strong electrostatic interaction with the nearest phosphate group. In addition, this amino group interacts by water bridges with other phosphate groups, sugar oxygen atoms, and oxygen, as well as with nitrogen atoms of the bases located along the length of the minor groove. 

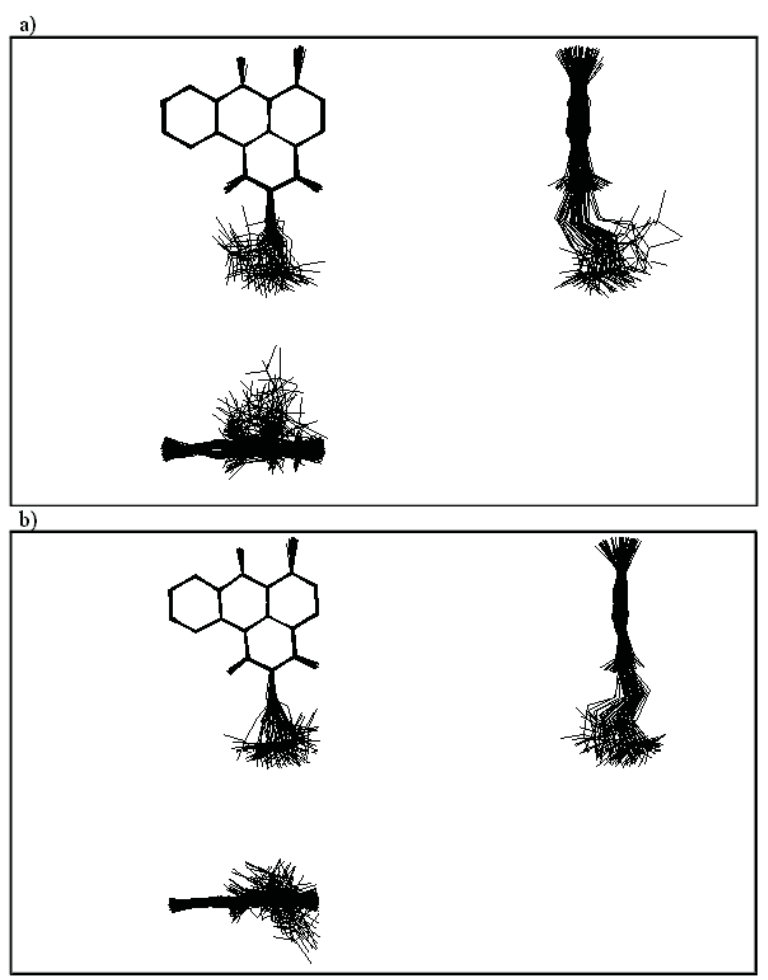

Figure 1. Orthogonal views of a composite of 40 snapshot geometries of PA 36: a) in water, and b) in the intercalation complex.

The acridine ring of the drug molecule in each snapshot has been fitted to its position in the first geometry. The dodecamer duplex, ions, and water molecules are omitted for clarity.

Some water bridges were also found between carbonyl oxygen OC17 and polar atoms of the surface of the minor groove. It is noteworthy that such bridges were not found for the second carbonyl oxygen attached to the pyrimidine ring.

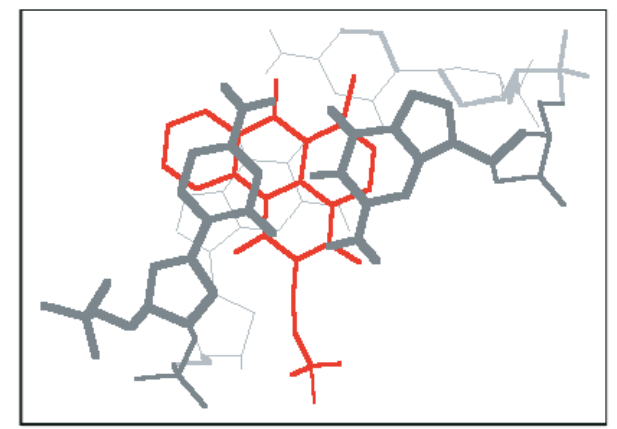

Figure 2. Orientation of PA 36 inside the intercalation cavity.

The intercalator molecule in red. Only two neighbor base-pairs are shown for clarity.

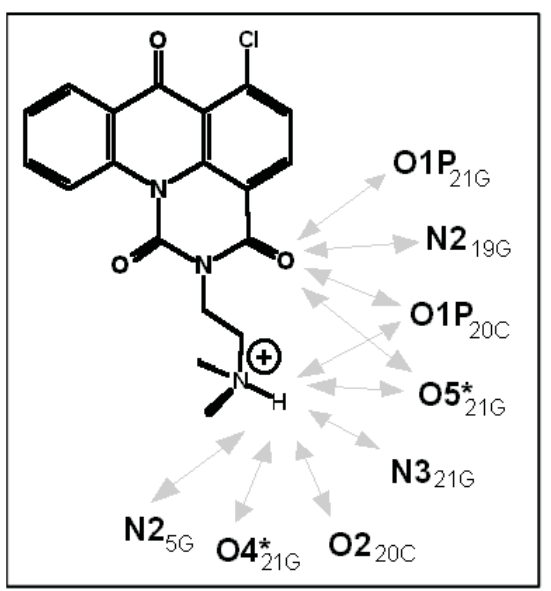

Figure 3. Water bridges (grey double arrows) between PA 36 molecule and the dodecamer observed during simulation.

Only interactions which exist by more than $10 \%$ of the simulation time are presented.

\section{PA 51}

The atoms forming the acridine part of the PA 51 ring system lie in one plane and during dynamic simulation in water, exhibit only small deviation from this plane, Fig. 4a. The pyrimidine ring $\mathrm{D}$ exhibits a slightly higher flexibility. The aromatic amino group in position 1 lies in the mean plane of the ring system. In such a geometry the hydrogen atom HNC1 could form an intramolecular hydrogen bond with carbonyl oxygen OC9. This bond was, in fact, detected in the dynamic trajectory of PA 51 in water. However, contrary to PA 36, the main part of the $N, N$-dimethylaminoethylamino side chain attached to $\mathrm{C} 1$ lies outside the mean plane of the ring system. This results from the geometrical state of the bond $\mathrm{NC1}-\mathrm{C} 1 \#$.

A very similar geometry of the PA 51 molecule was obtained from the trajectory of its intercalation complex (Fig. 4b).

The analysis of the geometry of the intercalation complex consisting of PA $\mathbf{5 1}$ and the dodecamer duplex revealed that long axis of the acridine ring system is practically perpendicular to the direction of hydrogen bonds between $6 \mathrm{C}$ and $19 \mathrm{G}$ (Fig. 5). The mean plane of 


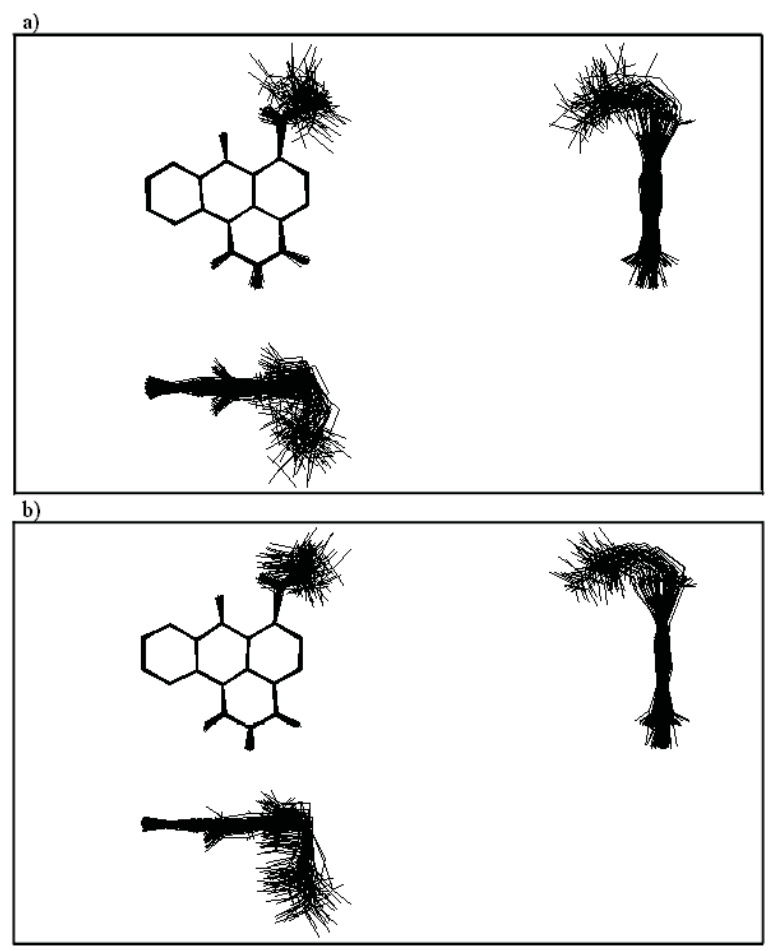

Figure 4. Orthogonal views of a composite of 40 snapshot geometries of PA 51: a) in water, and b) in the intercalation complex.

The acridine ring of the drug molecule in each snapshot has been fitted to its position in the first geometry. The dodecamer duplex, ions, and water molecules are omitted for clarity.

the ring system is parallel to the mean planes of the neighbor base-pairs. Moreover, the whole ring system is covered by neighbor base-pairs.

The side chain is oriented perpendicularly to the ring system and fits well into the interior

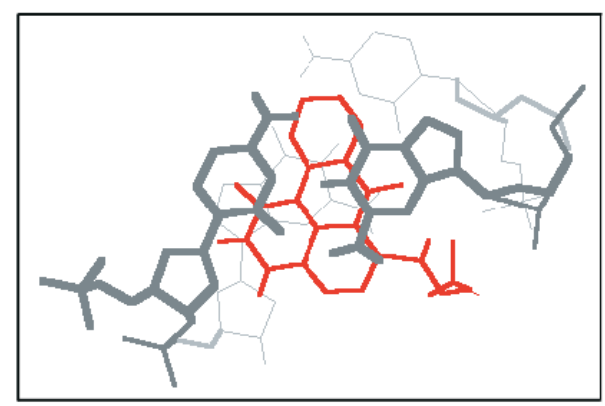

Figure 5. Orientation of PA 51 inside the intercalation cavity.

The intercalator molecule in red. Only two neighbour base-pairs are shown for clarity. of the minor groove. As a result, the protonated terminal amino group of the chain is located outside the intercalation cavity.

The absence of aminoalkyl chain on nitrogen N16, as well as the peculiar orientation of the PA 51 molecule inside the intercalation cavity opens the opportunity for formation of a large network of direct and indirect interactions between the DNA fragment and the intercalator (Fig. 6). This network comprises all polar atoms located in that part of the molecule, which is directed to the minor groove (Fig. 5). The hydrogen atom attached to N16

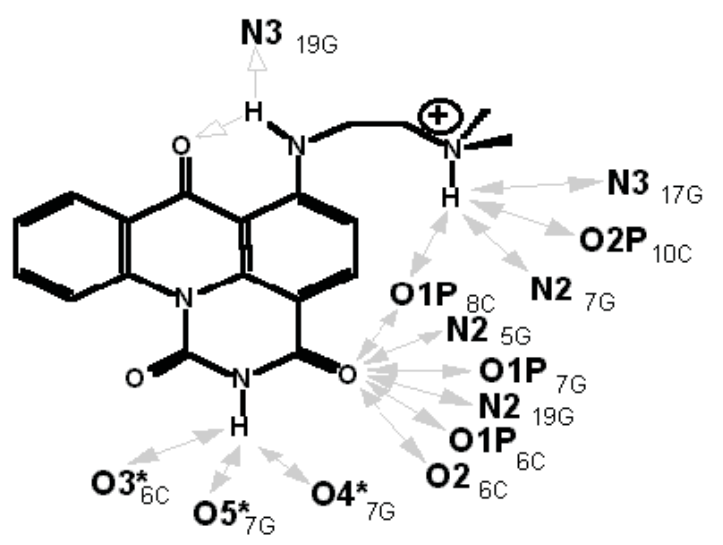

Figure 6. Hydrogen bonds (empty arrows) and water bridges (grey double arrows) between PA 51 molecule and the dodecamer observed during the simulation.

Only interactions which exist by more than $10 \%$ of the simulation time are presented.

interacts by water bridges with sugar oxygens from $6 \mathrm{C}$ and $7 \mathrm{G}$. The carbonyl oxygen OC17 is a centre of a network of water bridges. The interactions with phosphate groups of $6 \mathrm{C}, 7 \mathrm{G}$, and $8 \mathrm{C}$ form the main part of this network. However, the bridges between OC17 and base nitrogens from $5 \mathrm{G}$ and $19 \mathrm{G}$ are also observed.

The protonated terminal amino group forms its own network of water bridges which connect the side chain with phosphate groups of $8 \mathrm{C}$ and $10 \mathrm{C}$, as well as with base nitrogens from $7 \mathrm{G}$ and $17 \mathrm{G}$. 
The hydrogen HNC1 from aromatic amino group in position 1 forms a strong and relatively stable intramolecular hydrogen bond with acridinone oxygen OC9. This bond was observed during about $50 \%$ of the simulation period and stabilised the coplanar orientation of the amino group. Moreover, the same hydrogen atom forms an intermolecular hydrogen bond with N3 atom of $19 \mathrm{G}$ during $16 \%$ of the simulation.

\section{PA 39}

The PA 39 molecule may be treated as a superposition of the two molecules presented above. One may expect that the dynamic behaviour of this molecule could be also obtained by superposition of dynamics of PA 36 and PA 51. The analysis of the dynamic tra-
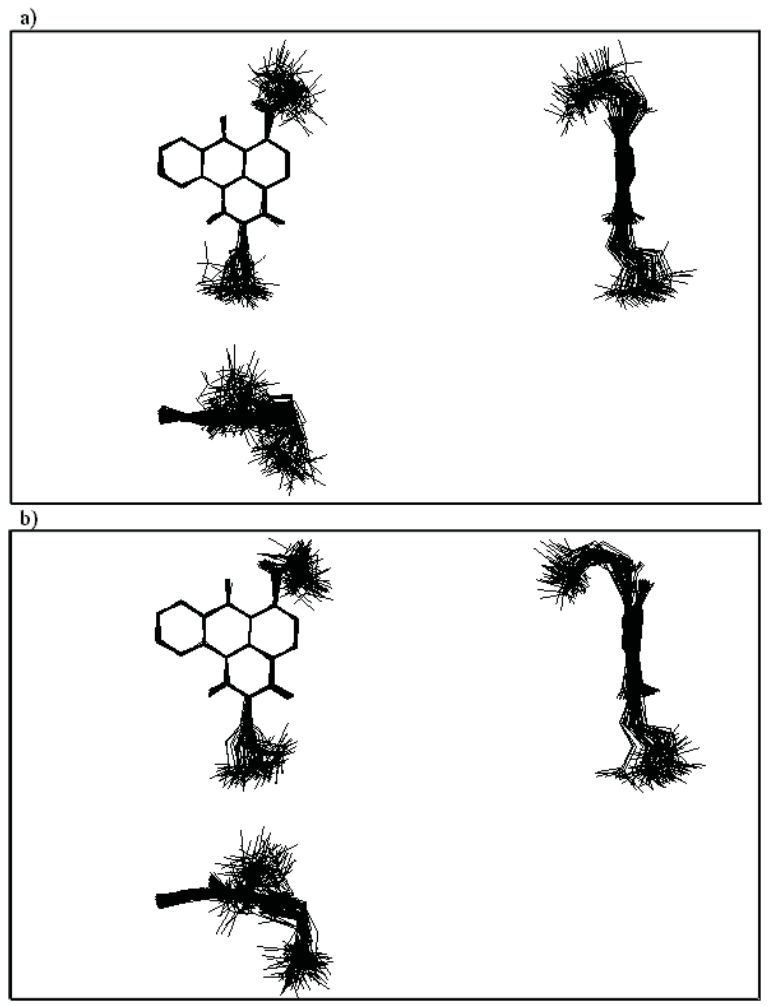

Figure 7. Orthogonal views of a composite of $\mathbf{4 0}$ snapshot geometries of PA 39: a) in water, and b) in the intercalation complex.

The acridine ring of the drug molecule in each snapshot has been fitted to its position in the first geometry. The dodecamer duplex, ions, and water molecules are omitted for clarity. jectory of PA 39 in water revealed that this supposition is reasonable (Fig. 7a). The side chain attached to N16 is located near the mean plane of the ring system, whereas the chain attached to $\mathrm{C} 1$ is bent out of the plane. The dynamics observed in water indicates that, in the PA 39 molecule a change in one side chain does not change the behaviour of the other.

The overall shape and dynamic behaviour of the PA 39 molecule which participates in the formation of the intercalation complex are very similar to what we have obtained from dynamic simulation in water (Fig. 7b). However, a more detailed comparison reveals some significant differences. The ring system of the molecule is not flat. Ring C (Scheme 2) is inclined with respect to the rest of the system by about $20^{\circ}$. In addition, the aromatic amino group in position 1 does not lie in the plane of ring $\mathrm{A}$.

The presence of those deformations indicates that the PA 39 molecule undergoes some particular tensions in the intercalation cavity. They may result from collision between requirements of the two side chains. The structure of the intercalation complex presented on Fig. 8 supports this supposition. The angle between the long axis of the acridine system and the direction of hydrogen bonds between $6 \mathrm{C}$ and $19 \mathrm{G}$ is about $45^{\circ}$. Moreover, the degree of intercalation is rather low.

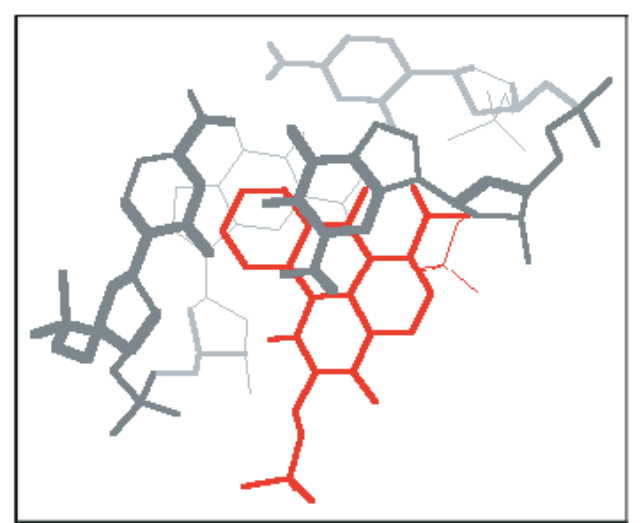

Figure 8. Orientation of PA 39 inside the intercalation cavity.

The intercalator molecule in red. Only two neighbor base-pairs are shown for clarity. 
Only rings $\mathrm{B}$ and $\mathrm{C}$ are covered by the neighbor base pairs. Rings A and D are located inside the minor groove. Non planar geometry of the base pairs $6 \mathrm{C} / 19 \mathrm{G}$ and $7 \mathrm{G} / 18 \mathrm{C}$ also indicates that the intercalation of the PA 39 molecule induces relatively large forces which deform the intercalator molecule, as well as the intercalation cavity.

As a result of shallow intercalation observed for PA 39 a relatively large part of the molecule is located in the minor groove. In this geometry of the intercalation complex both carbonyl oxygens of the pyrimidine ring can form hydrogen bonds with water molecules located in the minor groove. Some of these molecule form also hydrogen bonds with phosphate groups. As a result, a network of water bridges is formed (Fig. 9). The protonated, ter-

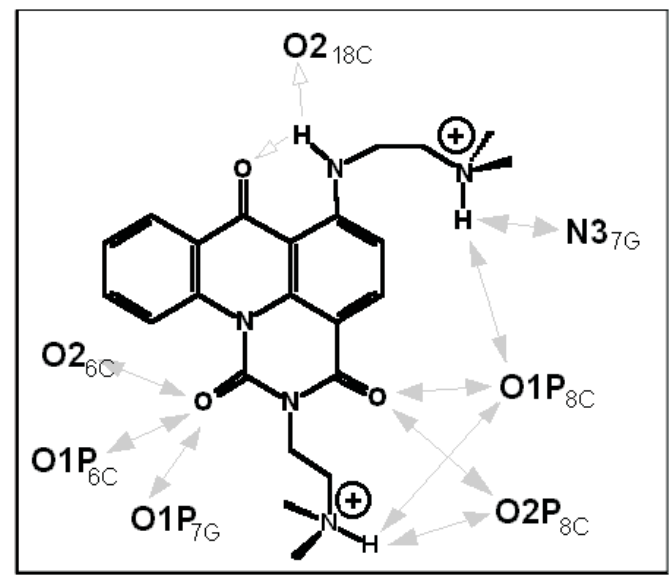

Figure 9. Hydrogen bonds (empty arrows) and water bridges (grey double arrows) between PA 39 molecule and the dodecamer observed during the simulation.

Only interactions which exist by more than $10 \%$ of the simulation time are presented.

minal amino groups from the side chains also participate in formation of this network. Moreover, the terminal, protonated amino group from the chain attached in position 1 forms a water bridge with $\mathrm{N} 3$ atom of $7 \mathrm{G}$. The hydrogen HNC1 forms not only an intramolecular hydrogen bond with OC9 but also a direct hydrogen bond with $\mathrm{O} 2$ atom of $18 \mathrm{C}$. All of these interactions stabilise the particular geometry of the intercalation complex formed by the PA 39 molecule.

\section{DISCUSSION}

It is interesting to note that the three derivatives of pyrimidoacridinetrione studied in this work exist in water in forms which seem to be ready for intercalation into DNA. Orientations as well as conformations of the side chains do not change significantly when the molecules form the intercalation complexes. This is particularly surprising if one keeps in mind our results of molecular dynamics simulations obtained for imidazoacridinones [31]. However, the conformation of the chain, as well as its orientation in relation to the ring system depend on the position of the attachment.

The $N, N$-dimethylaminoethyl chain attached to N16 atom (PA 36, Fig.1) adopts a nearly elongated conformation and is placed near the mean plane of the ring system. It is characteristic for this chain that its geometry is not affected by the addition of the second chain in position 6, PA 39 (Fig. 7). The $N, N$-dimethylaminoethylamino chain attached in position 6 (PA 51, Fig. 4) adopts a quite different conformation and orientation. The aromatic amino group is practically coplanar with the rings $\mathrm{A}$ and $\mathrm{B}$. In this geometry $\mathrm{HNC} 1$ hydrogen atom forms an intramolecular hydrogen bond with OC9. However, geometrical states of the bonds $\mathrm{NC} 1-\mathrm{C} 1 \#$ and $\mathrm{C} 1 \#-\mathrm{C} 2 \#$ decide that the rest of the chain is located perpendicularly to the ring system. The geometrical states of the same bond determine a bent conformation of the chain. As a result, the terminal, protonated amino group is located far from the mean plane of the ring system. The presence of an additional chain on N16 (PA 39, Fig. 7) atom does not affect the geometry and dynamics of this chain.

In another work [31] we have studied a series of imidazoacridinones. In three com- 
pounds from this series the $N, N$-diethylaminoethylamino chain is attached in the same position as the side chain in PA 51. A comparison of geometry of similar side chains attached to different ring systems may be very instructive. The geometry of the side chain of imidazoacridinones in water depends on the type of substituents in ring C. When the ring system was substituted by a hydroxyl or methyl moiety the side chain adopted, due to formation of intramolecular hydrogen bonds, a characteristic "closed" conformation. When a t-butyl group was attached to ring $\mathrm{C}$ of imidazoacridinone the "closed" conformation was not formed and the side chain adopted a similar, but not an identical, geometry with that observed for the side chain of PA 51 in water. It is noteworthy that the geometries of the two side chains are practically the same when the molecules studied participate in formation of the intercalation complex.

The results obtained in this study clearly show that, in the case of PAT derivatives, the position of side chain attachment determines:

$\checkmark$ i) the orientation of the intercalator ring system inside the intercalation cavity,

-ii) the type of interaction between the intercalator and DNA.

When the chain is attached to the N16 atom (PA 36) the long axis of the acridine ring system is parallel to the direction of inter-base hydrogen bonds between 6C and 19G (Fig. 2). This orientation is a result of:

- electrostatic attractive interaction between the protonated terminal amino group of the side chain and the nearest phosphate group of DNA,

the network of water bridges formed between this group and other phosphate groups, sugar oxygen atoms, and oxygen, as well as nitrogen atoms of the bases located along the length of the minor groove (Fig. 3). A characteristic feature of these bridges is their "vertical" direction.

When the chain is attached in position 1 (PA 51) the long axis of the acridine ring sys- tem is perpendicular to the direction of inter-base hydrogen bonds between $6 \mathrm{C}$ and 19G (Fig. 5). This orientation is a result of fitting of the side chain into the minor groove and is stabilised by the network of water bridges (Fig. 6). Unlike in the complex of PA 36, these bridges have rather horizontal directions. The direct hydrogen bond between $\mathrm{HNC} 1$ and $\mathrm{N} 3$ atom of $19 \mathrm{G}$ also stabilises this orientation.

It is noteworthy that if only one side chain is attached to the pyrimidoacridine skeleton the ring system intercalated between base-pairs is flat and parallel to their mean plane (Fig. 10). However, the geometry of the intercalation complex dramatically changes when the pyrimidoacridinetrione derivative e.g. PA 39, has two chains. The geometrical preferences of the chains are totally different and can not be fulfilled at the same time. The structure of the complex formed by such an intercalator exhibits a significant deformation. One may expect that such a deformed complex should be unstable. However, experimental data exhibit high stability of the PA 39/DNA intercalation complex. The experimental constant of the intercalation complex formation is about 10 times higher for PA 39 than for PA 36 [23]. Results of our dynamic simulations also suggest that the PA 39/dodecamer complex is stable. Changes in geometry of the complex were observed only during the thermalisation step when the deformed state of the intercalation cavity was formed. After that state was established no significant changes in geometry of the complex were observed during more than $100 \mathrm{ps}$ of the simulation (Fig. 10). This means that the increase of interaction energy between the duplex and the intercalator more than compensates for deformation energy. The PA 39/duplex complex is one of the examples that our intuitive conviction that any deformation of optimal geometry results in instability of the system is not always true.

The new, low-energetic type of the intercalation cavity which was observed in the present work opens interesting perspectives in the 


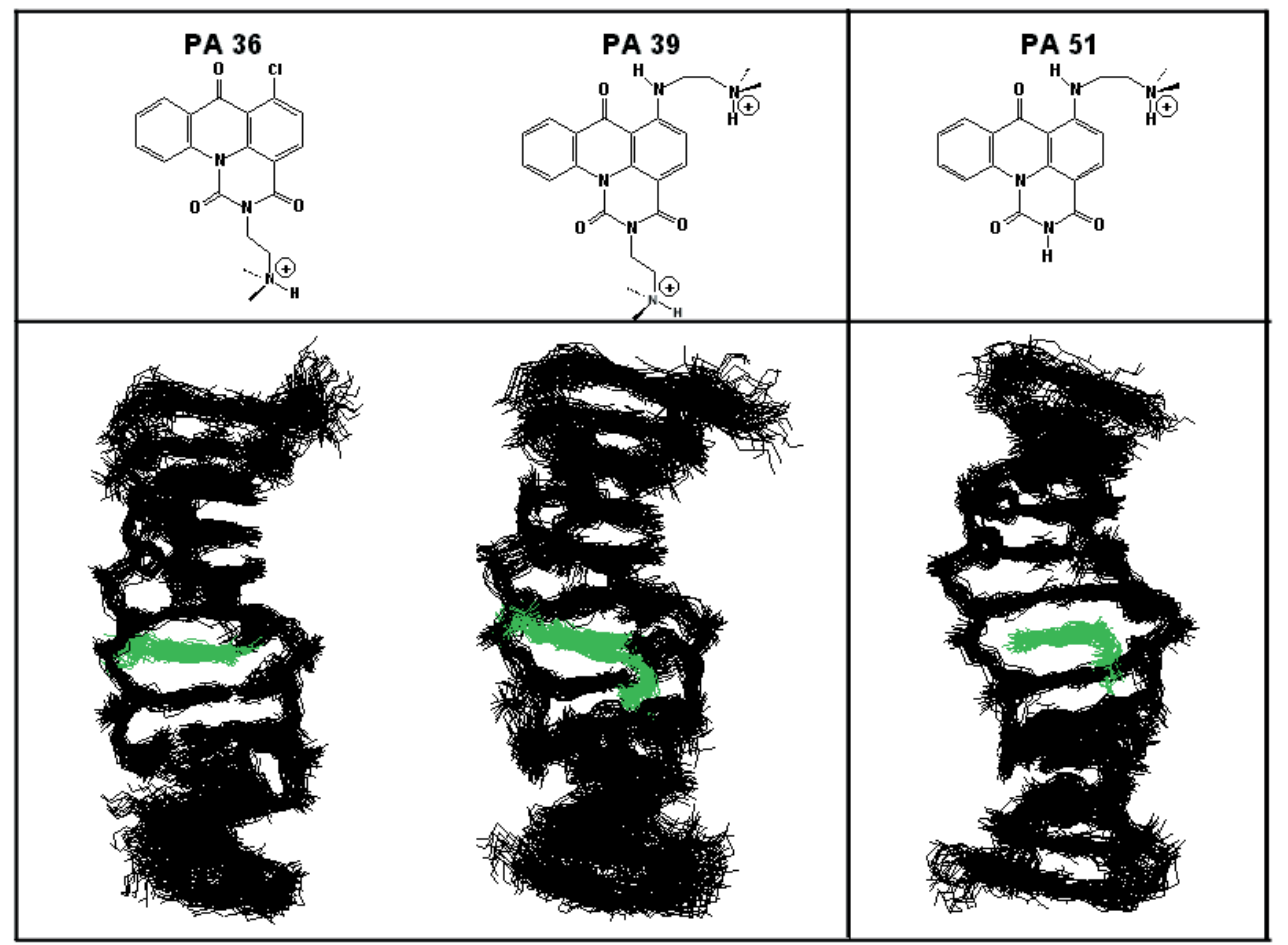

Figure 10. Composites of 40 snapshot geometries of PAT/dodecamer complexes.

Views from the minor groove side. The sugar $\mathrm{C} 1^{*}$ atoms of eight central base-pairs in each snapshot have been fitted to their positions in the first snapshot. The ions and water molecules are omitted for clarity.

field of designing new intercalating agents. More detailed analysis of forces responsible for the deformation of the intercalation cavity, as well as the mechanism of the deformation, will be subject of our further investigations.

\section{R E F E R E N C E S}

1. Cheng, C.C. \& Zee-Cheng, R.K.Y. (1983) The design, synthesis, and development of a new class of potential antineoplastic anthraquinones; in Progress in Medicinal Chemistry (Ellis, G.P. \& West, G.B., eds.) pp. 83-118 and references cited therein, Elsevier, Amsterdam.

2. Zee-Cheng, R.K.Y. \& Cheng, C.C. (1978) Antineoplastic agents. Structure-activity relationship study of bis(substituted aminoalkyloamino)anthraquinones. J. Med. Chem. 21, 291-294.
3. Zee-Cheng, R.K.Y., Podrebarac, E.G., Menon, C.S. \& Cheng, C.C. (1979) Structural modification study of bis(substituted aminoalkyloamino)anthraquinones. An evaluation of the relationship of the [2-[(2-hydroxyethyl)amino] ethyl]amino side chain with antineoplastic activity. J. Med. Chem. 22, 501-505.

4. Murdock, K.C., Child, R.G., Fabio, P.F., Angier, R.B., Wallace, R.E., Durr, F.E. \& Citarella, R.V. (1979) Antitumor agents. 1. 1,4Bis[(aminoalkyl)amino]9,10-anthracenediones. J. Med. Chem. 22, 1024-1030.

5. Durr, F.E. (1988) Biochemical pharmacology and tumor biology of mitoxantrone and ametantrone; in Anthracycline and Anthracenedione-Based Anticancer Agents (Lown, J.W., ed.) pp. 401-445, Elsevier, Amsterdam.

6. Cain, B.F. \& Atwell, G.J. (1974) The experimental antitumor properties of three congeners of the acridinyl methanesulphonanilide (AMSA) series. Eur. J. Cancer 10, 539-549. 
7. Denny, W.A., Baguley, B.C., Cain, B.F. \& Waring, M.J. (1983) Antitumor acridines; in Molecular Aspects of Anticancer Drug Action (Neidle, S. \& Waring, M.J., eds.) pp. 1-34, Verlag Chemie, Basel.

8. Showalter, H.D.H., Johnson, J.L., Werbel, L.M., Leopold, W.R., Jackson, R.C. \& Elslager, E.F. (1984) 5-[(Aminoalkyl)amino]-substituted anthra-[1,9-cd]-pyrazol-6(2H)-ones as novel anticancer agents. Synthesis and biological evaluation. J. Med. Chem. 27, 253-255.

9. Leopold, W.R., Nelson, J.M., Plowman, J. \& Jackson, R.C. (1985) Anthrapyrazoles. A new class of intercalating agents with high level, broad spectrum activity against murine tumors. Cancer Res. 45, 5532-5539.

10. Capps, D.B., Dunbar, J., Kesten, S.R., Shillis, J., Werbel, L.M., Plowman, J. \& Ward, D.L. (1992) 2-(Aminoalkyl)-5-nitropyrazolo[3,4,5$k l$ lacridines. A new class of anticancer agents. J. Med. Chem. 35, 4770-4778.

11. LoRusso, P., Wozniak, J., Polin, L., Capps, D., Leopold, W.R., Werbel, L.M., Biernat, L., Dan, M.E. \& Corbett, T.H. (1990) Antitumor efficacy of PD 115934 (NSC 366140) against solid tumors of mice. Cancer Res. 60, 4900-4905.

12. Showalter, H.D.H., Angelo, M.M., Berman, E.M., Kanter, G.D., Ortwine, D.F., RossKesten, S.G., Turner, W.R., Werbel, L.M., Worth, D.F., Elslager, E.F., Leopold, W.R. \& Shillis, J.L. (1988) Benzothiopyranoindazoles, a new class of chromophore modified anthracenedione anticancer agents. Synthesis and activity against murine leukemias. J. Med. Chem. 31, 1527-1539.

13. Atwell, G.J., Rewcastle, G.W., Baguley, B.C. \& Denny, W.A. (1987) Potential antitumor agents. 50. In vivo solid-tumor activity of derivatived of $N$-[2-(diethylamino)ethyl]acridine-4-carboxamide. J. Med. Chem. 30, 664-669.
14. Wakelin, L.P.G., Atwell, G.J., Rewcastle, G.W. \& Denny, W.A. (1987) Relationship between DNA-binding kinetics and biological activity for the 9-aminoacridine-4-carboxamide class of antitumor agents. J. Med. Chem. 30, 855-861.

15. Cholody, W.M., Martelli, S., Paradziej-Lukowicz, J. \& Konopa, J. (1990) 5-[(Aminoalkyl)amino]imidazo[4,5,1-de]acridin-6-ones as a novel class of antineoplastic agents. Synthesis and biological activity. J. Med. Chem. 33, 49-52.

16. Cholody, W.M., Martelli, S. \& Konopa, J. (1992) Chromophore-modified antineoplastic imidazoacridinones. Synthesis and activity against murine leukemias. J. Med. Chem. 35, 378-382.

17. Cholody, W.M., Martelli, S. \& Konopa, J. (1990) 8-Substituted 5-[(aminoalkylamino]$6 \mathrm{H}$-triazolo[4,5,1-de]acridin-6-ones as potential antineoplastic agents. Synthesis and biological activity. J. Med. Chem. 33, 2582-2586.

18. Antonini, I. \& Martelli, S. (1992) The synthesis of a new polycyclic heterocyclic ring system: Pyrimido[5,6,1-de]acridine. J. Heterocyclic Chem. 29, 471-473.

19. Antonini, I., Cola, D., Martelli, S., Cholody, W.M. \& Konopa, J. (1992) Pyrimidoacridine derivatives as potential antitumor agents. $I l$ Farmaco, Ed. Sci. 47, 1035-1046.

20. Antonini, I., Cola, D., Polucci, P., BontempsGracz, M., Borowski, E. \& Martelli, S. (1995) Synthesis and biological evaluation of dialkylaminoalkyl disubstituted pyrimidoacridinetriones, a novel group of antitumor, resistance reversing agents. J. Med. Chem. 38, 3282-3286.

21. Denny, W.A. (1989) DNA-Intercalating ligands as anticancer drugs: Prospects for future design. Anti-Cancer Drug Des. 4, 241-263.

22. Baguley, B.C. (1991) DNA Intercalating antitumor agents. Anti-Cancer Drug Des. 6, 1-35. 
23. Baginski, M., Polucci, P., Antonini, I., Borowski, E. \& Martelli, S. (1999) Binding free energy of selected anticancer compounds to DNA - theoretical calculations. Biophys. Chem. (in press).

24. Levitt, M. (1982) Computer simulations of DNA double helix dynamics. Cold Spring Harbor Symposia on Quant. Biol. XLVII, 251-262.

25. Cieplak, P., Rao, S.N., Grootenhuis, P.D.J. \& Kollman, P.A. (1990) Free energy calculations on base specificity of drug-DNA interactions: Application to daunomycin and acridine intercalation into DNA. Biopolymers 29, 717-727.

26. Creighton, S., Rudolph, B., Lybrand, T., Singh, U.C., Shafer, R., Brown, S., Kollman, P.A. \& Andrea, T. (1989) A combined 2D-NMR and molecular dynamics analysis of the structure of the actinomycin D:d(ATCGAT) 2 complex. J. Biomol. Struct. Dyn. 6, 929-969.

27. Langley, D.R., Doyle, T.W. \& Beveridge, D.L. (1991) The dyneimicin-DNA intercalation complex. A model based on DNA affinity cleavage and molecular dynamics simulations. $J$. Am. Chem. Soc. 113, 4395-4403.

28. Boehncke, M., Nonella, M., Schulten, K. \& Wang, A.H.-J. (1991) Molecular dynamics investigation of the interaction between DNA and dystamycin. Biochemistry 30, 5465-5474.

29. Herzyk, P., Neidle, S. \& Goodfellow, J.M. (1992) Conformation and dynamics of
drug-DNA intercalation. J. Biomol. Struct. Dyn. 10, 97-139.

30. Mazerski, J., Martelli, S. \& Borowski, E. (1998) The geometry of intercalation complex of antitumor mitoxantrone and ametantrone with DNA: Molecular dynamics simulations. Acta Biochim. Polon. 45, 1-11.

31. Mazerski, J. \& Muchewicz, K. (1999) The intercalation of imidazoacridinones into DNA induces conformational changes in their side chain. Acta Biochim. Polon. 47, 65-78.

32. VanGusteren, W.F., Billeter, S.R., Eising, A.A., Hunenberger, P.H., Kruger, P., Mark, E.A., Scott, W.R.P. \& Tiron, I.G. (1996) Biomolecular simulation: GROMOS96 manual and user guide. vdf Hochschulverlag AG, Zurich.

33. Ryckeart, J.P., Ciccotti, G. \& Berendsen, J.H.C. (1977) Numerical integration of the cartesian equations of motion of a system with constraints: Molecular dynamics of n-alkanes. J. Comput. Phys. 23, 327-341.

34. Subraminian, P.S., Ravishanker, G. \& Beveridge, D.L. (1991) Molecular dynamics of B-DNA including water and counterions. A 140-ps trajectory for d(CGCGAATTCGCG) based on the GROMOS force field. J. Am. Chem. Soc. 113, 5027-5040. 